\title{
LOS HAMMATOCERATINAE (AMMONITINA) DEL TOARCIENSE Y AALENIENSE EN LA CORDILLERA IBÉRICA Y CANTÁBRICA ORIENTAL. IMPLICACIONES PALEOBIOGEOGRÁFICAS
}

\author{
Gemma MARTÍNEZ' \\ ${ }^{1}$ Dpto. y UEI de Paleontología. Facultad de CC. Geológicas (UCM) e Instituto de Geología Económica (CSIC-UCM).28040- \\ Madrid.
}

\begin{abstract}
Martínez, G. 1996. Los Hammatoceratinae (ammonitina) del Toarciense y Aaleniense en la Cordillera Ibérica y Cordillera Cantábrica oriental. Implicaciones paleobiogeográficas. [The Toarcian and Aalenian Hammtoceratinae (ammonitina) in the Iberian Range and the Eastern Cantabrian Mountains. Paleobiogeographic implications]. Revista Española de Paleontología, $\mathbf{N}^{0}$ Extraordinario, 114-121. ISSN 0213-6937.
\end{abstract}

\begin{abstract}
One of the ammonite subfamilies present in the northern Spain Lias and Dogger materials is the Hammatoceratinae, whose record is mostly scarce proportionaly to the other groups. 43 species and 7 genera have been identified, with a distribution range from the Toarcian (Variabilis Zone, Illustris Subzone) to the Bajocian (Discites Zone). Discrimination between the Mesogean and the NW Europe areas characteristic elements is possible on account of the study of the morphologycal aspects and the identification of the differents taphonomical associations. The origin of the group might be found in some Mesogean forms, which were the origin of invasions towards platform areas. In most of the cases the registrated elements correspond to drifted shells whith more or less remote original regions in relation to the burial place; the minor occurrence corresponds to real pioneer individuals of some areas of the Iberian Basin and the Vasco-Cantabrian Basin during several episodes of the Insigne, Aalensis and Opalinum Zones.
\end{abstract}

Keywords: Cephalopods, Jurassic, Biostratigraphy, Associations, Spain.

\section{RESUMEN}

Los Hammatoceratinae constituyen una de las subfamilias de ammonoideos que están presentes en los materiales del Lias y del Dogger del Norte de España, con un registro escaso en relación al resto de los ammonoideos. Se han distinguido 43 especies y 7 géneros que se distribuyen desde la Zona Variabilis (Subzona Illustris) del Toarciense hasta la Zona Discites del Bajociense. El estudio de las diferentes morfologías y la identificación de las diferentes asociaciones tafonómicas, permiten reconocer en ellas elementos procedentes de áreas mesogeas y del NW de Europa. El origen de este grupo podría encontrarse en determinadas formas mesogeas, que pudieron invadir áreas de plataforma. En la mayoría de los casos los elementos registrados corresponden a conchas derivadas procedentes de regiones más o menos alejadas del lugar de enterramiento. Así mismo existen elementos que pudieron colonizar algunas áreas de la Cuenca Ibérica y de la Cuenca Vasco-Cantábrica, durante determinados episodios de las Zonas Insigne, Alensis y Opalinum.

Palabras clave: Cefalópodos, Jurásico, Bioestratigrafía, Asociaciones, España.

\section{INTRODUCCIÓN}

En los últimos años se han realizado estudios sobre los ammonoideos de la subfamilia Hammatoceratinae, en el Este y Norte de la Península Ibérica, que han quedado recogidos en diferentes monografías y artículos ya publicados (Ureta, 1985; Goy, et al., 1991; Martínez, 1992 a,b). En este trabajo se presentan los resultados obtenidos sobre este grupo, que permiten proponer una serie de precisiones sobre su distribución bioestratigráfica, clasificación taxonómica, interpretación filogenética, así como realizar observaciones e interpretaciones de carácter paleobiogeográfico. Por otra parte, se ha creído conveniente refigurar los holotipos de las especies nuevas propuestas en la Tesis doctoral de la autora del presente trabajo, Martínez (1992b), debido a la deficiente calidad de las reproducciones originales.
Los ejemplares estudiados proceden de diferentes secciones de la Cordillera Ibérica y de la Cordillera Cantábrica oriental (Fig. 1). Aunque en su mayor parte fueron recogidos durante la realización de los trabajos de campo correspondientes a la citada Tesis doctoral, se han revisado también los ejemplares procedentes de diferentes colecciones de otros autores (Goy, 1974; Ureta, 1985; etc). El análisis detallado de los muestreos realizados, pone de manifiesto que los Hammatoceratinae son relativamente escasos en relación a otros grupos de ammonoideos. Por lo general no suponen más del $3 \%$ del total y sólo en determinados episodios muy puntuales representan el 30 o el $40 \%$ de los ammonoideos registrados.

Desde el punto de vista taxonómico, se han distinguido 43 especies incluídas en 7 géneros; que, por orden de aparición son: Geczyceras Martínez, Hammatoceras Hyatt, Bredyia Buckman, Planammatoceras Buckman, Euaptetoceras 


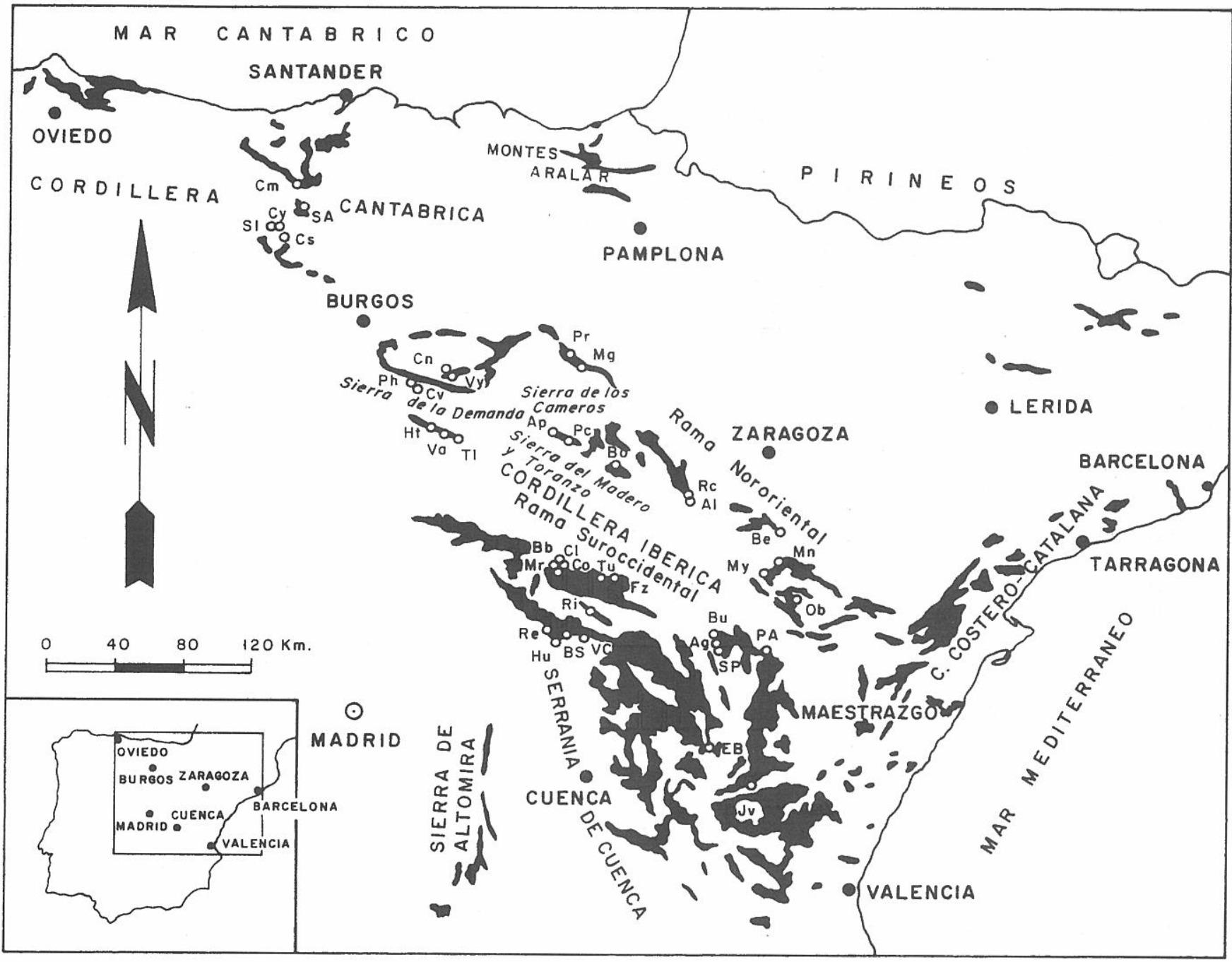

Figura 1. Afloramientos jurásicos y localización de los cortes estudiados en las Cordilleras Ibérica y Cantábrica Oriental: Cm: Camino; Cy: Cillamayor; SA: San Andrés; Cs: Camesa; Sl: Salinas de Pisuerga; Cn: Canales; Ph: Piedrahita de Nuño; Vy: Villavelayo; Cv: Castrovido; Pr: Préjano; Mg: Muro de Aguas; Ht: Hontoria; Va: Vadillo: Tl: Talveila; Ap: Aldealpozo; Pc: Pinilla del Campo; Bo: Borobia; Rc: Ricla; Al: La Almunia de Doña Godina; Be: Belchite; Mn: Moneva; My: Moyuela; Ob: Obón; Bb: Balbacil; Mr: Maranchón; Cl: Clares; Co: Codes; Tu: Turmiel; Fz: Fuentelsaz; Ri: Ribarredonda; Re: Renales; Hu: Huertahernando; BS: La Buenafuente del Sistal; VC: Villar de Cobeta; Bu: Bueña; Ag: Aguatón; SP: Sierra Palomera; PA: Perales de Alfambra; EB: Embalse de San Blas; Jv: Javalambre (Según Martínez, 1992b).

Buckman, Parammatoceras Buckman y Eudmetoceras Buckman.

\section{DISTRIBUCIÓN BIOESTRATIGRÁFICA}

En la Cordillera Ibérica, los Hammatoceratinae (Fig. 2) se extienden desde la Subzona Illustris del Toarciense hasta la Zona Discites del Bajociense. Se aprecian 5 intervalos con una mayor abundancia relativa de especímenes: 1) Zona Variabilis y parte inferior de la Zona Thouarsense, 2) Zona Insigne (Subzona Insigne)-Zona Pseudoradiosa (Subzona Levesquei), 3) Zona Aalensis (Subzona Aalensis)-Zona Opalinum (Subzona Comptum), 4) Zona Murchisonae (Subzona Bradfordensis p.p.) y 5) Zona Murchisonae (Subzona Bradfordensis p.p.)-Zona Concavum. Por otra parte, existen dos lagunas registráticas: 1)
Zona Thouarsense (Subzona Thouarsense-Subzona Fascigerum) y 2) Zona Pseudoradiosa (Subzona Pseudoradiosa). En la Cordillera Cantábrica Oriental, debido al escaso número de ejemplares obtenidos, los datos no son lo suficientemente significativos para poder establecer con precisión los límites de los intervalos citados, a partir de la Zona Murchisonae.

La Subzona Insigne del Toarciense se caracteriza por contener una gran diversidad taxonómica, con una relación indivíduos/especie del género Geczyceras relativamente baja; por el contrario el género Hammatoceras, presenta menor número de especies pero con un alto número de indivíduos por especie. A partir de la Zona Aalensis y durante el Aaleniense el registro se hace cada vez más esporádico y discontínuo, excepto entre la Subzona Aalensis y la Subzona Comptum, donde se encuentra el género Bredyia de forma relativamente constante y contínua. En la Zona Murchisonae las especies más frecuentes pertenecen al género Planamma- 


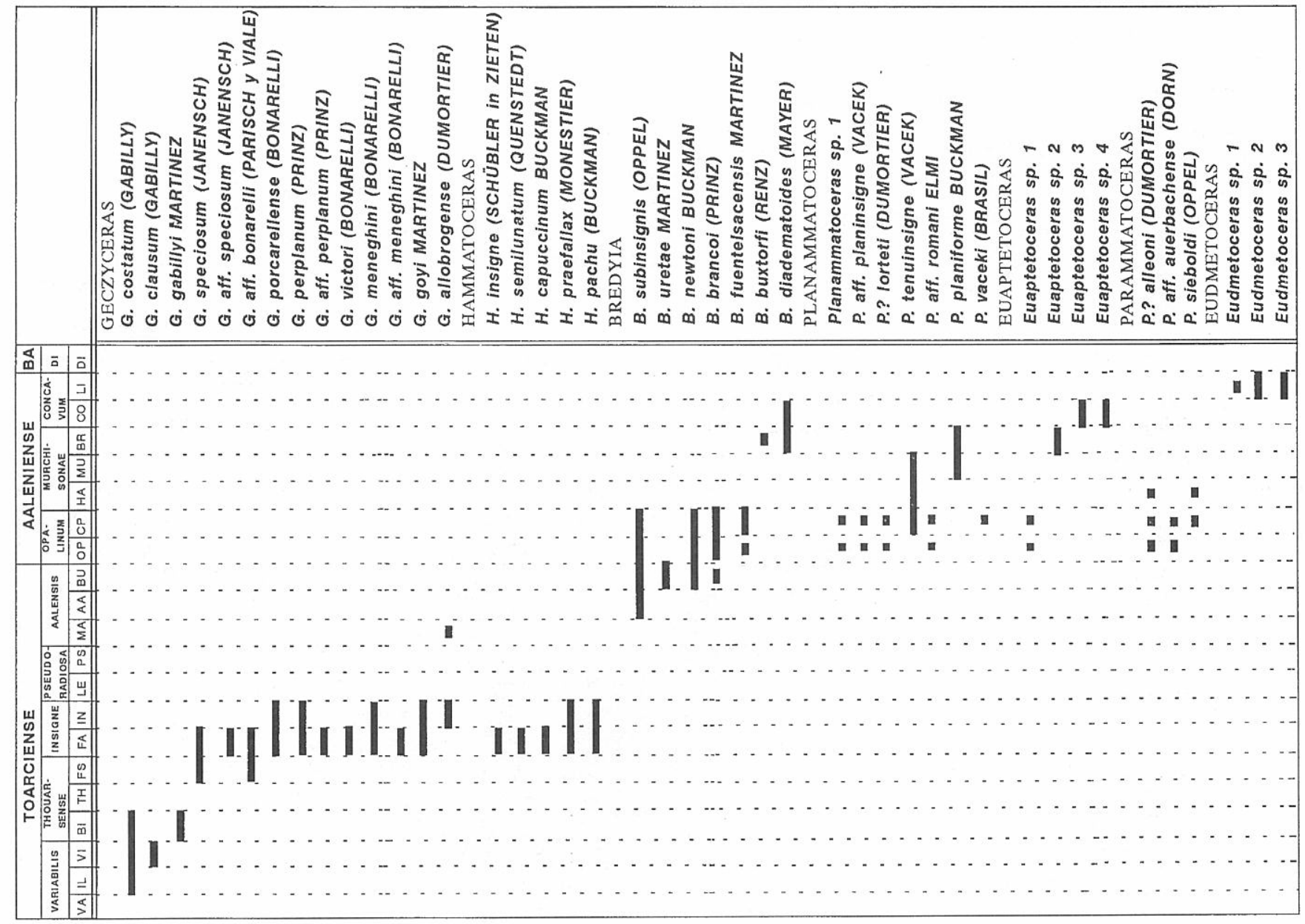

Figura 2. Distribución bioestratigráfica de los taxones identificados. (Modificado de Martínez, 1992b).

toceras, mientras que el resto de los elementos obtenidos tiene una presencia fortuita.

\section{CARACTERES MORFOLÓGICOS}

Los Hammatoceratinae engloban a ammonoideos con conchas que tienen distinto grado de involución, pudiendo variar de evolutas a involutas, con secciones comprimidas subovaladas, subredondeadas, subrectangulares, subojivales, etc. Presentan carenas netas, en general poco elevadas, ombligos de profundidad variable y la ornamentación es generalmente gruesa, habitualmente con nudos y costillas sinuosas o rectas. A pesar de esta gran variabilidad morfológica, existe un carácter distintivo que es la línea de sutura (Fig. 3); ésta es en general muy recortada, con lóbulo E de longitud variable, el L bien desarrollado y el conjunto de lóbulos umbilicales retraídos y oblicuos.

Teniendo en cuenta las características morfológicas de las líneas de sutura, se pueden diferenciar dos grupos de Hammatoceratinae:

- Uno que posee suturas muy recortadas, con L de tronco tabular, lóbulos auxiliares fuertemente retraídos y oblicuos entre sí y E largo, al que corresponde el género Geczyceras (Martínez, 1992b) (Fig. 3b; Lám. I, fig. 2a).

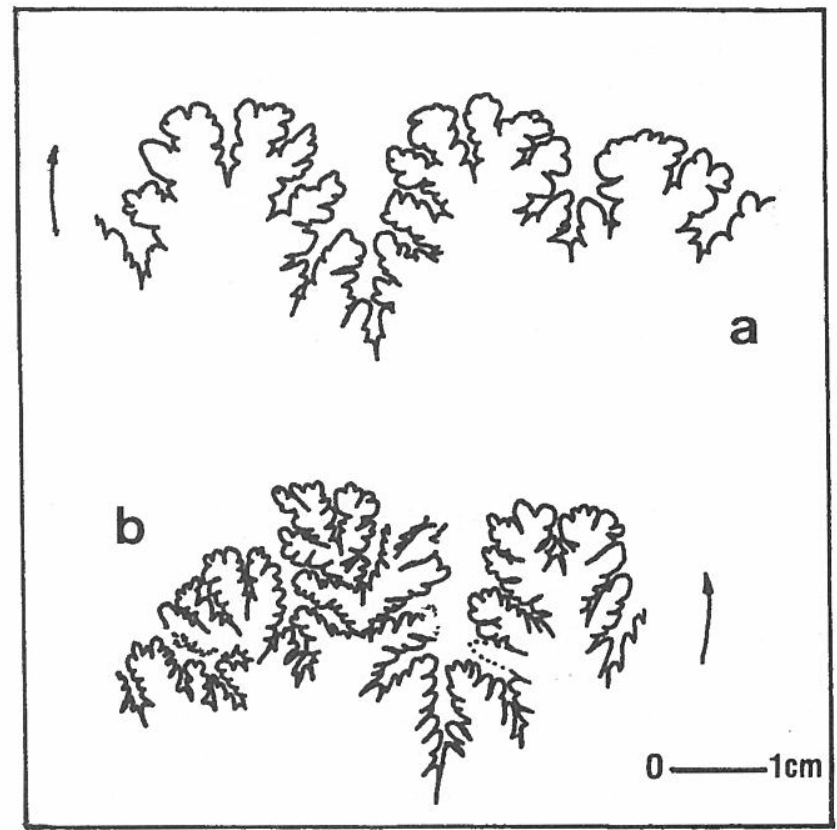

Figura 3. Línea de sutura. a. Hammatoceras capuccinum Buckman. Ej. Fz14/24; H= 84,9 mm; b. Geczyceras victorii (Bonarelli). Ej. Fz16/25; H= 51,2 mm. 
- Otro que posee suturas más simples, L de tronco triangular y grueso, lóbulos auxiliares menos retraídos y E de menor longitud, al que corresponde el género Hammatoceras (Fig. 3a; Lám. I, fig. 1a).

Morton (1975) propone un índice de complejidad sutural a partir de la longitud del lóbulo L y su anchura máxima; aplicándolo a formas de Hammatoceras y Geczyceras observamos que en éste último, los valores están comprendidos entre el $30 \%$ y el $51 \%$, mientras que en Hammatoceras se distribuyen en una banda estrecha y de valores más bajos (15\% a 26\%) (ver Martínez, op. cit., b; p. 202, fig. 40).

El grupo que presenta suturas de alta complejidad se caracteriza además por poseer enrollamientos evolutos, secciones subovaladas o subrectangulares y ombligos amplios y poco profundos. Este conjunto de caracteres ha sido interpretado por autores como Tintant et al. (1982) como adaptaciones a formas de vida pasiva y con capacidad para los movimientos verticales. Dentro de este grupo también están incluídos los géneros del Aaleniense: Eudmetoceras, Planammatoceras, Euaptetoceras y Parammatoceras, si bien los tres últimos poseen enrollamientos involutos y secciones triangulares y afiladas, lo que ha sido interpretado como adaptaciones a una forma de vida nectobentónica, cerca del fondo en aguas tranquilas.

Los elementos de estos géneros, tanto en el Toarciense como en el Aaleniense, se encuentran, en las series de las cuencas estudiadas, representados por un número de especies alto y con un número de individuos bajo, lo que hace pensar que no corresponden a faunas autóctonas.

El grupo de suturas menos recortadas y de complejidad sutural menor, que incluye también al género Bredyia, poseen enrollamientos más involutos, con secciones ojivales y afiladas y ombligos estrechos y profundos. Este conjunto de caracteres ha sido interpretado como adaptaciones a medios someros y más o menos confinados.

Los representantes de estos dos géneros, se encuentran en determinados niveles de las series estudiadas con un número de especies relativamente bajo, pero con un número de individuos alto.

\section{INTERPRETACIÓN FILOGENÉTICA}

A la vista de los datos obtenidos y de acuerdo con Arkell (1957), Geczy (1966), etc., el origen de los Hammatoceratinae se encontraría en determinadas faunas autóctonas de áreas mesogeas, correspondientes a la Zona Bifrons o a la parte inferior de la Zona Variabilis. Teniendo en cuenta este supuesto, es posible que, a partir de formas primitivas como los (Hammatoceras?) Praerycites Venturi, 1981 se produzcan diferentes emigraciones hacia áreas de plataforma, que den lugar, en la Subzona Illustris, a los Hammatoceratinae más antiguos registrados en la Cordillera Ibérica, Cordillera Cantábrica Oriental y en el Centro-Oeste de Francia: Geczyceras costatum (Gabilly, 1973), G. clausum (Gabilly, 1973) y G. gabillyi Martínez, 1992 (Lám. II, fig. 1). Posteriormente, a partir de G. bonarellii (Parisch y Viale, 1906) especie típica de áreas mesogeas o submesogeas, pudo originarse $G$. speciosum (Janensch, 1902) especie con una distribución geográfica muy amplia y que tiene su primer registro en la Cordillera Ibérica en la Subzona Fallaciosum. Esta especie,
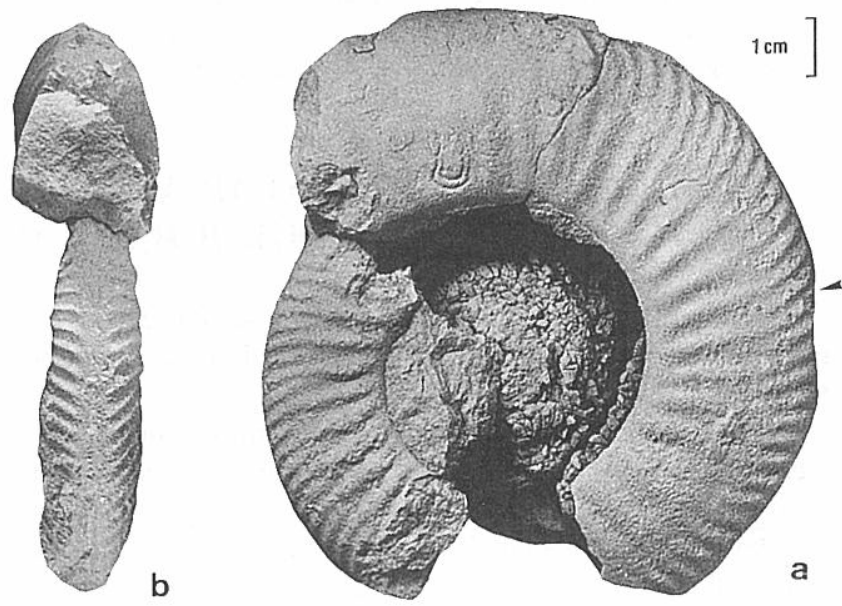

Figura 4. Geczyceras goyi Martínez. Ej. Ow7/1. Holotipo. Fragmocono y parte de la cámara de habitación. Zona Insigne. Obón. a: vista lateral; b: vista ventral. (La flecha indica el comienzo de la cámara de habitación).

pudo dar lugar en áreas de plataforma al género Hammatoceras y en particular a las especies $H$. insigne, $H$. semilunatum (Quenstedt, 1885) у H. pachu (Buckman, 1921). Por otro lado $G$. speciosum también pudo dar lugar a $H$. capuccinum Buckman, 1921 y al grupo de Geczyceras porcarellense (Bonarelli, 1899), G. perplanum (Prinz, 1904), G. allobrogense (Dumortier, 1874), G. victorii (Bonarelli, 1899), G. meneghinii (Bonarelli, 1899) y G. goyi Martínez, 1992 (Fig. 4) que a su vez, en áreas del Tethys, darían lugar a los Planammatoceras. Probablemente a partir de $G$. perplanum y $G$. allobrogense se produjo una nueva diferenciación de formas típicas de áreas de plataforma, originándose el género Bredyia, con morfotipos típicos de la Cuenca Ibérica como B. uretae Martínez, 1992 (Lám. II, fig. 2) y B. fuentelsacensis Martínez, 1992 (Lám. II, fig. 3). Uno de los representantes de este género, B. brancoi (Prinz, 1904) o alguna forma afín, pudo dar lugar a los Parammatoceras y estos a los Eudmetoceras. La relación filogenética de Euaptetoceras y Parammatoceras resulta bastante más difícil de justificar a partir de los datos obtenidos en las áreas estudiadas, ya que el material es muy escaso y discontínuo. En otras áreas donde el registro es más

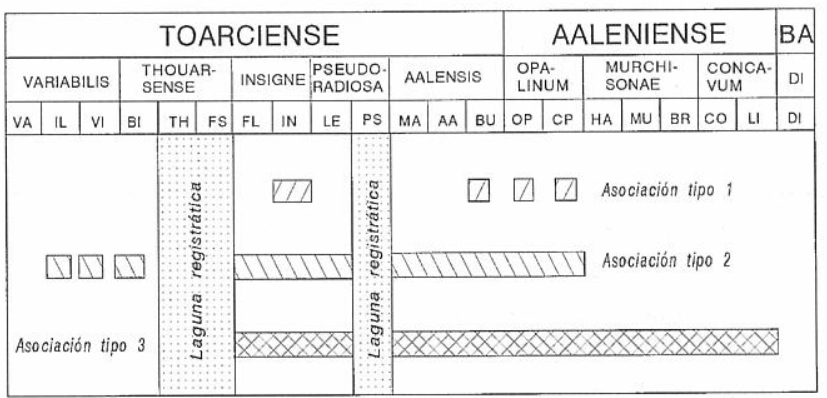

Figura 5. Tipos y distribución de Asociaciones registradas de Hammatoceratinae en el Toarciense y Aaleniense de las cuencas estudiadas (modificado de Martínez, 1992b). 
abundante, Hungría por ejemplo, ciertos autores como Geczy (1966) indican que pueden proceder de formas de origen mediterráneo como Csernieyceras Geczy, 1966.

\section{ASOCIACIONES TAFONÓMICAS E IMPLICACIONES PALEOBIOGEOGRÁFICAS}

Desde el punto de vista tafonómico se han distinguido las siguientes asociaciones (Fig. 5) (cf. Fernández-López, 1985):

Las Asociaciones de tipo 3 son las más generalizadas, en ellas, casi todos los restos corresponden a indivíduos adultos o de talla grande, que probablemente corresponden a conchas derivadas o flotadas desde áreas muy alejadas. Incluirían sobre todo a ciertos elementos de Geczyceras en las Zonas Insigne y Pseudoradiosa del Toarciense y a Eudmetoceras, Euaptetoceras y Parammatoceras en el Aaleniense.

En las Asociaciones de tipo 2 predominan las conchas de indivíduos de tamaño semejante, en general grande y son muy escasos o faltan los individuos jóvenes. Probablemente han sufrido deriva necroplantónica desde otras áreas más o menos alejadas. Incluirían a determinados elementos de Geczyceras en las Zonas Variabilis, Insigne y Pseudoradiosa del Toarciense y a ciertas Bredyia y Planammatoceras en la Zona Aalensis del Toarciense, y en la Zona Opalinum del Aaleniense.

En las Asociaciones de tipo 1 predominan los indivíduos jóvenes que pueden ser monoespecíficos, con presencia de pares dimórficos. Probablemente corresponden a una sola asociación faunística que vivió cerca del lugar de enterramiento. Los únicos taxones cuyos elementos probablemente corresponden a este tipo de asociación son Hammatoceras insigne (Schlübler in Zieten, 1830) en determinados episodios de la Subzona Insigne y con dudas Bredyia subinsignis (Oppel, 1956), en determinados episodios de las Subzonas Buckmani y Comptum.

En definitiva, si tenemos en cuenta los tipos de asociaciones tafonómicas identificadas podría decirse que las condiciones ambientales tanto en la Cuenca Ibérica como en la Cuenca Vasco-Cantábrica durante el Toarciense superior y el Aaleniense, no fueron en general favorables para la colonización y el desarrollo de los Hammatoceratinae, de manera que las conchas correspondientes a la mayoría de los elementos reconocidos han debido experimentar un transporte post-morten desde otras áreas.

\section{AGRADECIMIENTOS}

La realización de esta síntesis, derivada de mi Tesis doctoral, ha sido posible gracias al Prof. Dr. Antonio Goy y a la Dra. Soledad Ureta que dirigieron la citada Tesis; así mismo, también agradezco al Dr. Sixto Fernández sus numerosos consejos y sugerencias a lo largo de la realización de la misma. A D. Eulogio Martín mi reconocimiento por la realización de las fotografías.

Este trabajo ha sido realizado en el marco del proyecto PB91-0383 DGICYT.

\section{BIBLIOGRAFÍA}

Arkell, W. 1957. Mesozoic Ammonoidea. Mollusca 4. Cephalopoda Ammonoidea. In: Treatise on Invertebrate Paleontology. (Ed. R. C. MOORE). L. The University of Kansas Press, 1-490.

Fernández-López, S. 1985. El Bajociense de la Cordillera Ibérica. Tesis doctoral, Dpto. Paleontología, Universidad Complutense de Madrid, 1-850 pp.

Geczy, B. 1966. Ammonoides jurassiques de Csernye, Moutagne Bakony, Hongrie - Part. I (Hammatoceratidae). Geologica Hungarica, series Palaeontogica, 34, 275 pp.

Goy, A. 1974. El Lías de la mitad Norte de la Rama Castellana de la Cordillera Ibérica. Tesis Doct. Fac. Geología, Univ. Complutense, 3 t., XV + 940 p. Resumen: Publ. Depart. Paleont., Univ. Compl. Madrid, 14, 35 pp.

Goy, A. ; Martínez, G. y Ureta, S. 1991. "The succession of Hammatoceratinae in the Aalenian of Northern Spain". In: N. MORTON, Ed.; Conference on Aalenian and Bajocian Stratigraphy. Isle of Skye. Birbeck College. University of London, 49-63.

Martínez, G. 1992a. Extension stratigraphique du genre Hammatoceras dans le Lias de la Cordillère Iberique. Les Cahiers de l'Université Catholique de Lyon, Série Sciences, 5, 37-50.

Martínez, G. 1992b. Hammatoceratinae (Ammonitina) del Toarciense superior y Aaleniense en la Cordillera Ibérica. Col.

\section{Lámina I.}

1. Hammatoceras capuccinum Buckman. Ej. Fz14/24. Fragmocono. Zona Insigne (Subzona Insigne). Fuentelsaz. a: vista lateral; b: vista ventral.

2. Geczyceras victorii (Bonarelli). Ej. Fz16/25. Fragmocono. Zona Insigne (Subzona Insigne). Fuentelsaz. a: vista lateral; b: vista ventral.

Escala gráfica: $1 \mathrm{~cm}$

\section{Lámina II.}

1. Geczyceras gabillyi Martínez. Ej. Ph411/1. Holotipo. Fragmocono. Adulto. Zona Thouarsense. Piedrahita de Nuño. a: vista lateral; b: vista ventral.

2. Bredyia uretae Martínez. Ej. 2My82/1. Holotipo. Fragmocono y parte de la cámara de habitación. Zona Aalensis (Subzona Buckmani). Moyuela. a: vista lateral; b: vista ventral.

3. Bredyia fuentelsacensis Martínez. Ej. Fz148/2. Holotipo. Fragmocono. Zona Opalinum (Subzona Comptum). Fuentelsaz. a: vista lateral; b: vista ventral.

Escala gráfica: $1 \mathrm{~cm}$. 
Lámina I
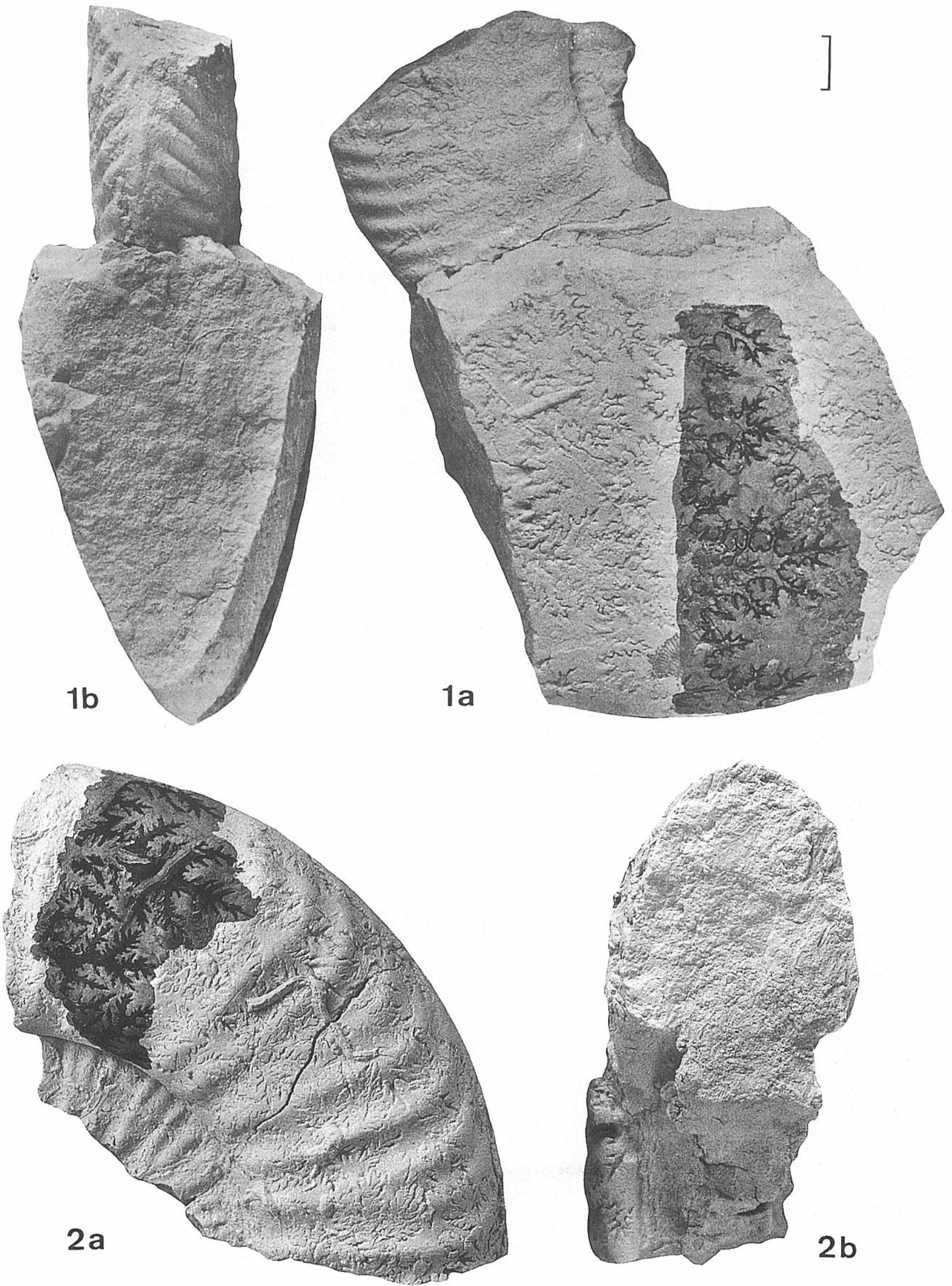


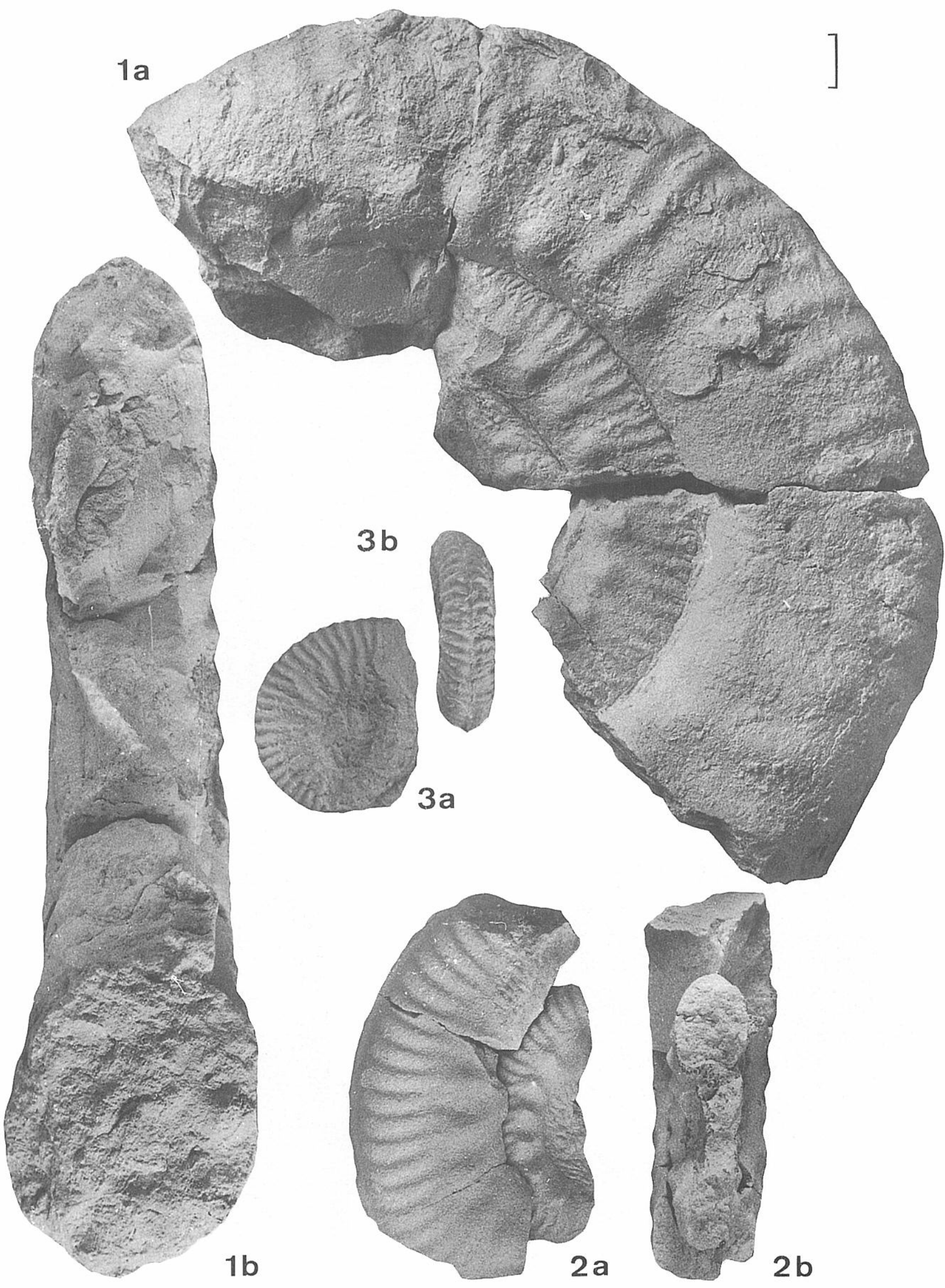


Tesis Doctorales, Universidad Complutense de Madrid, 374/92, $331 \mathrm{pp}$.

Morton, N. 1975. Bajocian Sonniidae and other Ammonites from Western Scotland. Palaeontology, 18, 41-91.

Tintant, H.; Marchand, D. et Mouterde, R. 1982. Relations entre les milieux marins et l'évolution des Ammonoïdes: les radiations adaptatives du Lias. Bull. Soc. géol. France, 7, XXIV, 951-961.
Ureta, M. S. 1985. Bioestratigrafía y Paleontología (Ammonitina) del Aaleniense en el Sector Noroccidental de la Cordillera Ibérica. Col. Tesis Doctorales, Universidad Complutense de Madrid, 158/85, $452 \mathrm{p}$.

Trabajo recibido: 1 de febrero, 1995 Trabajo aceptado: 20 de octubre, 1995 